Faculdade de Ciências Econômicas UFRGS
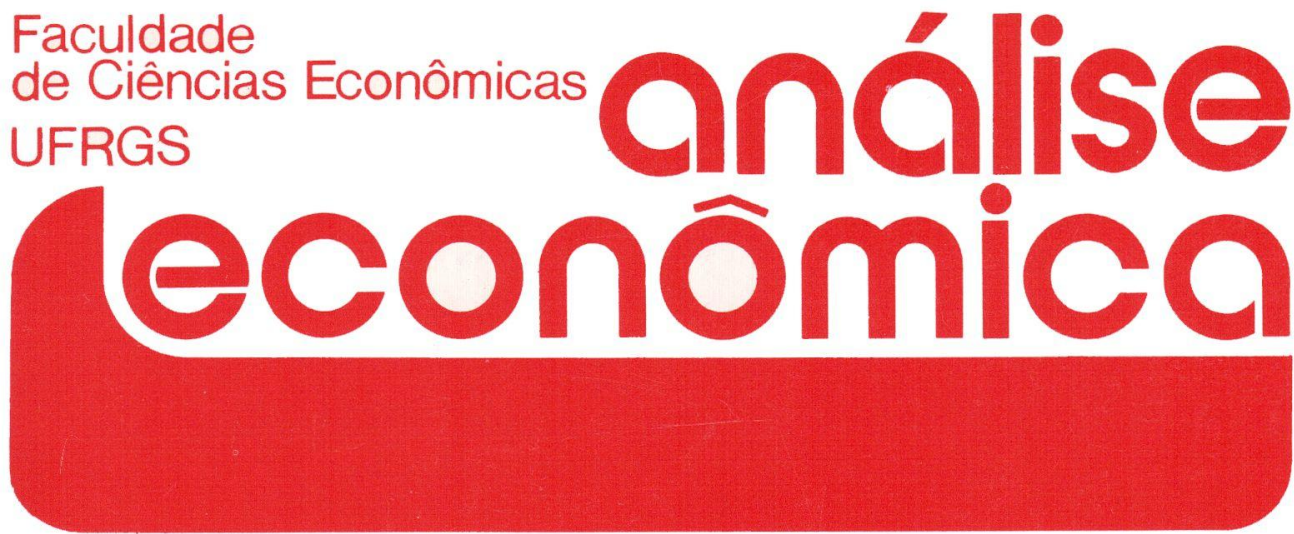

- STATE AND THE LIBERALIZATION OF THE BRAZILIAN ECONOMY

Carlos Alberto Longo

- MOEDA E CRÉDITO NA ECONOMIA BRASILEIRA: UM MODELO COM VETORES DE CORREÇÃO DE ERROS

Rolando M. Guzmán

- baSES PARA UM NOVO MOdELO DE POLítica FISCAL, PARA O BRASIL

Flávio Riani

- POR QUE OS SINOS DOBRAM? CONFLITO TRABALHISTA E SALÁRIOS DO MAGISTÉRIO PÚBLICO DO RIO GRANDE DO SUL, 1974-1991

Carlos Henrique Horn

Roberto Balau Calazans

- A POLÍtica MONETÁRIa E AS TAXAS de JUROS NO PLANO COLLOR

Lauro Lobo Burle

- DIFUSÃo E TRANSFERÊNCIA DA TECNOLOGIA DE PRODUÇÃO DE CIMENTO NO BRASIL

Maria Cristina Pereira de Melo

- SISTEMA FINANCEIRO BRASILEIRO: UMA PROPOSTA dE disCuSSÃo

Luiz Felipe Serpa

- TEORIA ECONÓMICA DO CASAMENTO E DO Divórcio

Giácomo Balbinotto Neto

- RESENHA

VARGAS: O CAPITALISMO EM CONSTRUÇÃO, DE PEDRO C. D. FONSECA

João Rogério Sanson

- LIVROS RECEBIDOS

Claudine Saldanha César
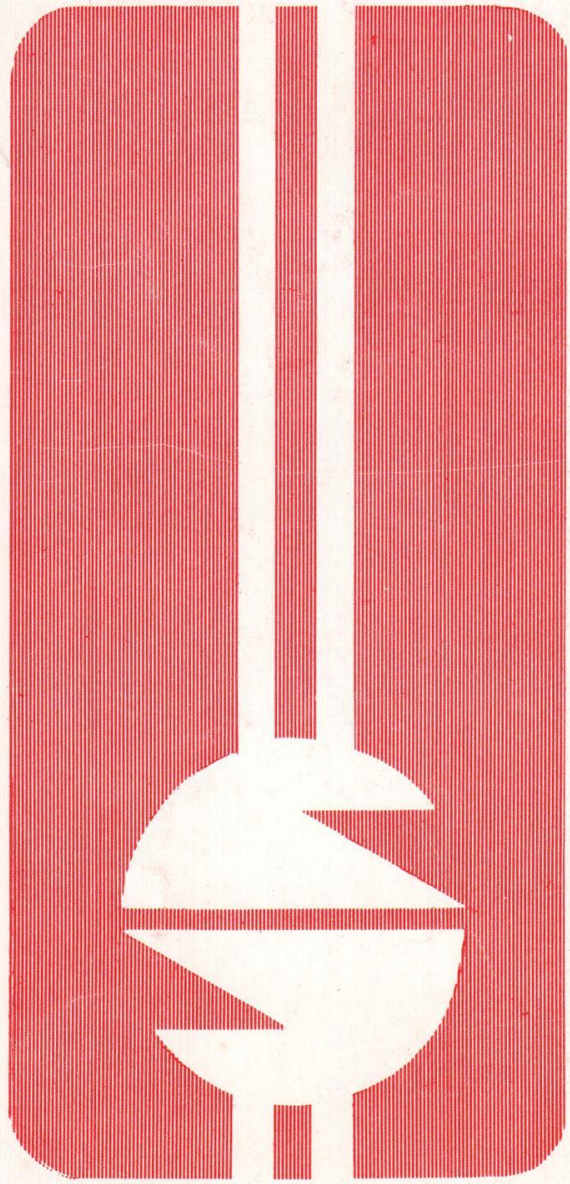
UNIVERSIDADE FEDERAL DO RIO GRANDE DO SUL

Reitor: Prof. Hélgio Henrique Casses Trindade

FACULDADE DE CIÉNCIAS ECONÔMICAS

Diretora: Prof ${ }^{a}$ Yeda Rorato Crusius

CENTRO DE ESTUDOS E PESQUISAS ECONÔMICAS

Diretor: Reinaldo Ignacio Adams

DEPARTAMENTO DE CIENNCIAS ECONÔMICAS

Chefe: Prof. Fernando Ferrari Filho

CURSO DE PÓS-GRADUAÇÃO EM ECONOMIA

Coordenador: Prof. João Rogério Sanson

CURSO DE PÓS-GRADUAÇÃO EM ECONOMIA RURAL

Coordenador: Prof. Juvir Luiz Mattuella

CONSELHO EDITORIAL: Achyles Barcelos da Costa, Aray Miguel Feldens, Atos Freitas Grawunder, Carlos Augusto Crusius, ErnaniHickmann, Fernando Ferrari Filho, João Rogério Sanson, Juvir Luiz Mattuella, Maria Imilda da Costa e Silva, Nali de Jesus de Souza, Nuno Renan Lopes de Figueiredo Pinto, Otília Beatriz Kroeĩ Carrion, Otto Guilherme Konzen, Paulo Alexaridre Spohr, Pedro Cezar Dutra Fonseca, Reinaldo Ignacio Adams, RobertsCamps Moraes, Valter José Stülp, Yeda Rorato Crusius, David Garlow (Wharton Econometrics Forecasts Association, E.U.A.), Edgar Augusto Lanzer (UFSC), Eleutério F. S. Prado (USP), Fernando Holanda Barbosa (FGV/RJ), Gustavo Franco (PUC/RJ), Joaquim Pinto de Andrade (UiNB), Juan H. Moldau (USP), Werner Baer (Univ. de Illinois, E.U.A.)

COMISSÃO EDITORIAL: Atos Freitas Grawunder, Reinaldo Ignacio Adams, Pedro Cezar Dutra Fonseca, Roberto Camps Moraes.

EDITOR: Nali Jesus de Souza

SECRETARIA: Maria Ivone de Mello (normalização), Vanete Ricacheski (revisão de textos)

FUNDADOR: Prof. Antônio Carlos Santos Rosa

Os materiais publicados na Revista Análise Econômica são de exclusiva responsabilidade dos autores. É permitida a reprodução total ou parcial dos trabalhos, desde que seja citada a fonte.

Aceita-se permuta com revistas congêneres. Aceitam-se, também, livros para divulgação, elaboração de resenhas ou recensões.

Toda correspondência, material para publicação (vide normas na $3^{a}$ capa), assinaturas e permutas devem ser dirigidos ao seguinte destinatário:

PROF. NALI DE JESUS DE SOUZA

Revista Análise Econômica

Av. João Pessoa, 52

90040-000 - PORTO ALEGRE (RS), BRASIL

Telefones: (051) 228.1633 - 224.6022 - Ramais 3348 ou 3440

FAX: (051) 225.1067 


\title{
MOEDA E CRÉDITO NA ECONOMIA BRASILEIRA: UM MODELO COM VETORES DE CORREÇÃO DE ERROS ${ }^{1}$
}

\author{
Rolando M. Guzmán
}

\section{SINOPSE}

Este artigo investiga a relaçāo entre a moeda, o crédito e o produto agregado, à luz da experiência brasileira no período 1980/1989. Um elemenuio essencial do estudo é a estimação de um modelo com Vetores de Correção de Erros (VEC), o que permite a identificação das relaçōes entre as variáveis no curto e no longo prazo, ao contrário das metodologias convencionais que analisam exclusivamente as relaçōes no curto prazo.

O texto inicia com um estudo das propriedades estocásticas das diferentes séries, prestando atenção particular às suas ordens de integração. Posteriormente, o modelo é estimado através do método de máxima verossimithança proposto por Johansen (1989) e Johansen \& Juselius (1990) Finalmente, a estimativa é utilizada para analisar o padrāo temporal de impulsos e respostas entre as variáveis

$\mathrm{Um}$ resultado relevante é o de que as flutuações do produto têm sido causadas predominantemente por choques monetários, com os choques de caráter crediticio tendo uma importância menor.

\section{INTRODUÇÃO}

Geralmente, as flutuações dos agregados riionetários são consideradas uma influência importante no comportamento do produto e dos níveis de preço da economia brasileira. Por conseguinte, o controie do meio circulante é costumeiramente visto como um objetivo intermediário através do qual se espera controlar, dentro de certos limites, os momentos do produto.

Se bem que a existência de um vínculo significativo entre c comportamento do produto e dos agregados monetários dificilmente poderia ser negada, diversos

1 Elaborado com base na dissertação de mestrado Moeda e Crédito na Economia Brasileira: Um Modelo com Vetores de Corrậāo de Erros,defendida em 1991, no Curso de Pos-Graduaçāo em Economia da Universidade Federal do Rio Grande do Sul. Tradução do espanhol feita por Marcelo Kuelle, bolsista de Iniciação Científica do CNPq.

ANÁLISE ECONÔMICA

ANO 10 $\mathrm{N}: 18$ SETEMBRO/92 P. 25.50 
indicadores sugerem que tal relação não foi particularmente estável no decorrer da década anterior. Considere, por exemplo, o comportamento da velocidade de circulação da moeda, medida como o quociente entre a oferta monetária e um índice do Produto Industrial. Este quociente não mostra somente uma clara tendência decrescente, como também mudanças significativas em seus níveis de variação.

\section{FIGURA I (a) \\ TRAJETÓRIA TEMPORAL DA RELAÇÃo \\ MOEDA/PRODUTO}

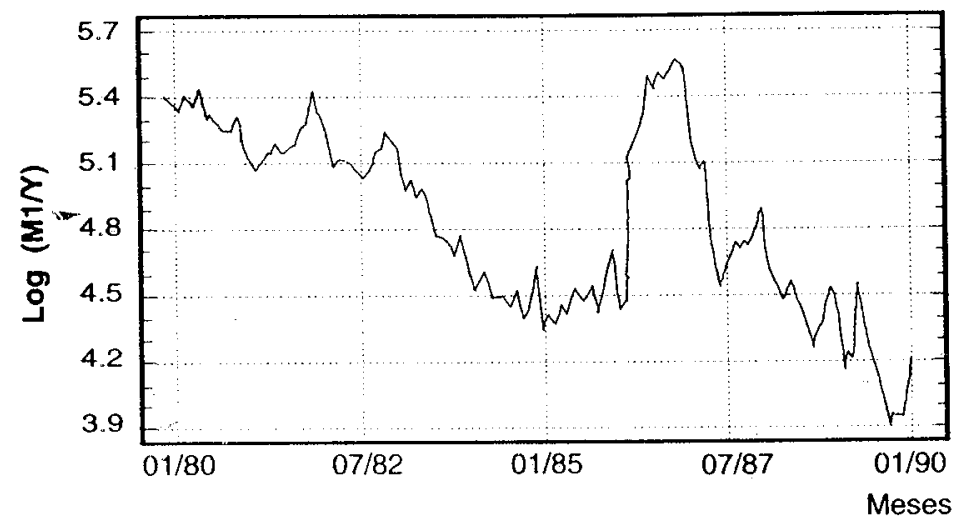

TABELA 1

RAZÃO MOEDA/PRODUTU ${ }^{\text {(a) }}$

INDICADORES ESTATÍSTICOS

\begin{tabular}{lllll}
\hline & $1980: 1 /$ & $1983: 1 /$ & $1986: 1 /$ & $1980: 1 /$ \\
& $1982: 12$ & $1985: 12$ & $1986: 12^{\text {(b) }}$ & $1989: 12$ \\
\hline MÉDIA & 5,220 & 4,640 & 5,260 & 4,830 \\
DESVIO PADRÃO & 0,114 & 0,225 & 0,390 & 0,416 \\
COEF. DE VARIAÇÃO & 0,022 & 0,048 & 0,070 & 0,086 \\
\hline
\end{tabular}

(a) Razão entre MI mensal, deflacionado pelo Índice de Preços ao Consumidor, e of f́ndice de Produto Industrial Real, calculado pelo IBGE.

(b) Período do Plano Cruzado. 
Na realidade, a instabilidade da relação moeda/produto não se limita ao caso brasileiro, sendo também uma característica de outras economias ocidentais. Nas últimas décadas, este fenômeno tem intrigado os econometristas, os quais têm tido grandes dificuldades para encontrar uma função estável da demanda por moeda. Nocontexto brasileiro, esta experiência se reflete na sempre presente discussãoem torno da possível quebra da demanda por moeda. ${ }^{2}$

Quais são as causas da instabilidade na velocidade da moeda na economia brasileira? Várias explicações poderiam ser apresentadas. ${ }^{3} \mathrm{Em}$ particular, alguns autores destacam a influência de outras variáveis financeiras, cuja exclusão poderia representar o elo perdido do problema. Os defensores desta posição sugerem, especificamente, a inclusão do crédito como uma variável relevante, cuja exclusão poderia ser responsável pela aparição de resultados inesperados ou confusos. ${ }^{4}$ A importância desta observação deve ser imediata: sé a influência do crédito sobre o produto agregado é particularmente poderosa, uma estratégia de política que incorpore a formação contida naquela variável deve ser preferível a uma estratégia centrada exclusiva (ou preferencialmente) no controle da oferta monetária. Este trabalho analisa a inter-relação entre oproduto, a moeda e o crédito na experiência brasileira recente. Especificamente, nosso objetivo consiste em comparar a importância dos choques creditícios e monetários nas flutuações dowproduto real, desde o ponto de vista das análises de séries temporais.

2 No início do século. Fisher (192.3) apresenta um primeiro estudo observando a instabilidade da relaçāo moeda/ produto. Goldfeld (1976) e Scadding \& Judd (1982) resumem a discussäo dos anos setenta. enquanto Poole (1988) apresenta discussöes mais recentes Cardoso (1981). Ross: (1988) e Vals Pereira (1990) represcntam trabalhos interessantes sobre a experiência brasileira

3 Uma explicação, não analisada neste trabalho. destaca a influência das inovaçōes - econômicas e tecnológicas - experimentadas pelo sistema financeiro brasileiro durante os últimos anos. Assim mesmo, outra explicação alternativa poderia estar baseada no papel das expectativas em um contexto de políticas instáveis e ambiente econômico mutável, características próprias da economia brasileira dos anos oitenta. Sc admitimos racionalidade nas expectativas, os agentes reformularão suas decisōes em função do rīßime vigente. Neste caso, a instabilidade das principais relaçōes seria um resultado esperado, em lugar de um resultado surpreendente.

4 As origens desta proposição são certamente antigas. voltando-se até os trabalhos de Fisher (1933) e Gurley \& Shaw (1955). Mais recentemente, a defesa do crédito como variável transcendente teve novo impulso a partir dos trabalhos de Mishkin (1976) e Bernanke (1983), nos quais se tentava explicar distintos períodos da economia norte-americana através de elementos não-monetários. Esta corrente destaca a fúnção do crédito no processo de realização dos planos nacionais do gasto agregado, função que está relacionada, mas não é idêntica nem subordinada, ao processo de geração de oferta monetária. Guzmán (1990) oferece maiores detalhes sobre este assunto. 


\section{FIGURA 2 (a) \\ TRAJETÓRIA TEMPORAL DA RELAÇÃo CREDITO/PRODUTO}

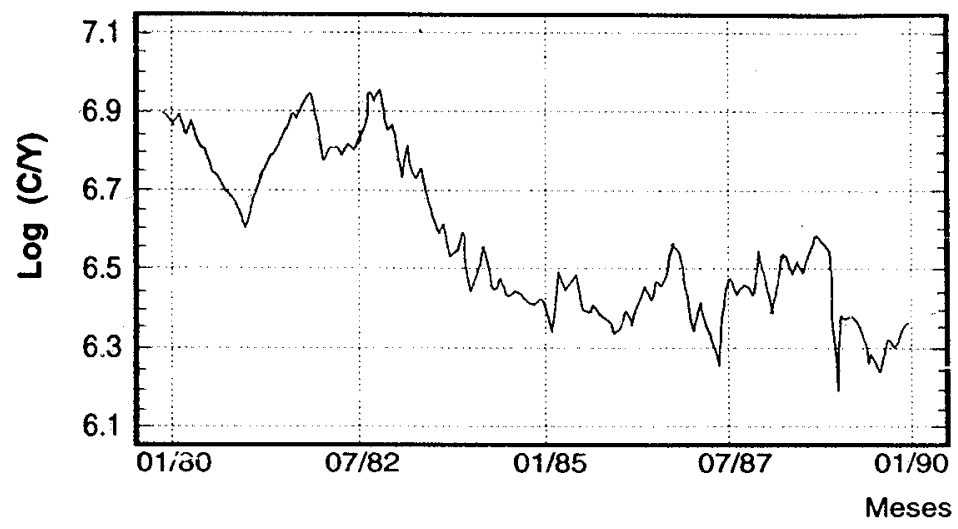

TABELA 2

RAZÃO CRÉDITO/PRODUTO ${ }^{(a)}$

INDICADORES ESTATÍSTICOS

\begin{tabular}{lllll}
\hline & $1980: 1 /$ & $1983: 1 /$ & $1986: 1 /$ & $1980: 1 /$ \\
& $1982: 12$ & $1985: 12$ & $1986: 12^{(b)}$ & $1989: 12$ \\
\hline MÉDIA & 6,800 & 6,530 & 6,440 & 6,573 \\
DESVIO PADRÂO & 0,085 & 0,140 & 0,079 & 0,194 \\
COEF. DE VARIAÇÃO & 0,013 & 0,022 & 0,011 & 0,030 \\
\hline
\end{tabular}

(a) Razão entre Crédito ao Setor Privado, deflacionado pelo Índice de Preços ao Consımidor, e o Indice de Produto Industrial Real, calculado pelo IBGE.

(b) Período do Plano Cruzado.

A exposição seguinte está dividida em seis seções. A seguiìda seção apresenta a especificação inicial do modelo econométrico utilizado. A terceira seção apresenta, de maneira extremamente concisa, a metodologia de estimação empregada. Na quarta seção, são estimadas as condições de equilíbrio de longo prazo entre as variáveis, reinterpretando o modelo básico como um modelo de Vetores de Correção de Erros (VEC). Mais tarde, a quinta seção discute a dinâmica do modelo fora das condições de equilíbrio, procurando determinar os padrões de impulsos e respostas no decorrer do tempo. Finalmente, a sexta seção resume as conclusões. 


\section{ESPECIFICAÇÃO INICIAL DO MODELO}

Tomemos como ponto de partida a representação auto-regressiva do modelo a ser estimado:

$$
\begin{aligned}
& X_{t}=\Pi_{1} X_{t-1}+\ldots+\Pi_{12} X_{t-12}+\vee D_{c t}+\xi D_{v t}+\mu+\varepsilon_{t} \\
& E\left(\varepsilon_{t}\right)=0 \\
& E\left(\varepsilon_{1} \varepsilon_{j}^{\prime}\right)= \begin{cases}\Sigma & i=j \\
0 & i \neq j\end{cases}
\end{aligned}
$$

(1)

onde $\mu, v$ e $\xi$ são vetores de dimensão $(4 \times 1)$ cujos elementos são parâmetros constantes, $\varepsilon_{t}$ é um vetor $(4 \times 1)$ de perturbações aleatóricas e $\Pi_{i}(i=1,2, \ldots 12)$ são matrizes formadas por parâmetros fixos..$^{5} \mathrm{D}_{\mathrm{ct}}$ e $\mathrm{D}_{\mathrm{vt}}$ são variáveis dummies destinadas a captar os efeitos dos Planos Cruzadoe Verão: $D_{\mathrm{ct}}$ vale 0 normalmente c 1 no período 1982:2/1987:2; $\mathrm{D}_{\mathrm{vt}}$ vale 1 no intervalo 1989:1/1989:12 e 0 nos demais períodos.

Por último $\chi_{̨}$ é um vetor com as seguintes variáveiis:

M: Oferta Monetária Real (M1)

C: Volume Real do Crédito ao Setor Privado

Y: Î́ndice da Produção Real

R: Taxa nominal Juros ${ }^{6}$

De forma mais clara, o modelo também pode ser descrito pela expressão alternativa:

(2)

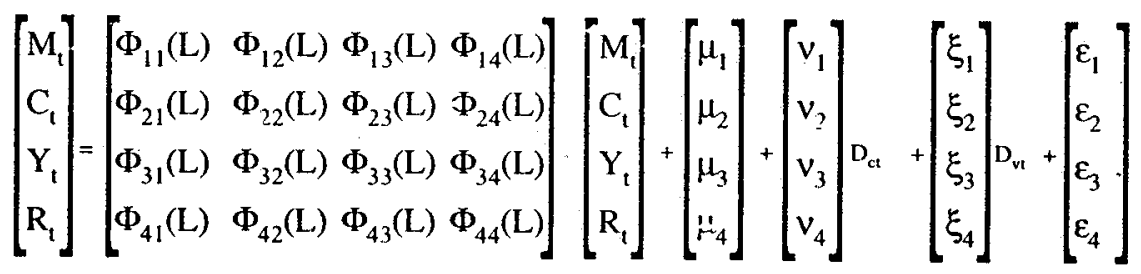

5 A estrutura de 12 defasagens implícita nesta definição das matrizes foi escolhida através de diversos testes $\mathrm{Fe}$ pelo Crédito de Informaçōes de Akaike (AIC).

6 Uma descrição mais precisa da contrapartida empírica das variáveis oferecidas em uma nota posterior. 
onde o elemento típico da primeira matriz de coeficientes, $\Phi_{\mathrm{ij}}(\mathrm{L})$, vem dado pela expressão:

$$
\Phi_{i j}(L)=\underset{n=0}{\sum^{12}} a_{n} L^{n}
$$

Já neste ponto, duas observações são convenientes. Em primeiro lugar, deve ser lembrado que o ordenamento das variáveis em um modelo vetorial pode ser sumamente importante. Nesta classe de modelos, a primeira variável do vetor é considerada, implicitamente, como independente das perturbações contemporâneas das demais variáveis e, por conseguinte, a menos que as covariâncias dos demais distúrbios possam ser depreciadas, a decomposição tas variâncias dos erros de predição será afetada pelo ordenamento adotado. A inclusão de M1, no lugar de agregados mais amplos, pretende justamente diminuir a incerteza no ordenamento adequado das variáveis, partindo do pressuposto razoável deque M1 éo agregado monetário que melhor poderia satisfazer a hipótese de independ contemporànea das demais variáveis do modelo. Por outro lado, dado que nosso objetivo se concentra na relação do produto com os agregados monetários e creditícios, a inclusão da taxa de juros parece indispensável, tendo em vista os resultados encontrados em outros estudos, onde as conclusões são sensíveis à inclusão ou à exclusão desta variável.

Como complemento, deve ser notado que todas as variáveis - exceto a taxa de juros - aparecem em termos reais. Portanto o modelo pode ser interpretado como um estudo das relações entre as variáveis depois de se descontar os efeitos sobre os preços.

Nosso primeiro objetivo consiste na estimação deste modelo. Especialmente, desejamos estimar os vetores de co-integração do modelo - interpretando tais vetores como relações de equilíbrio de longo prazo - e as relações dinâmicas entre suas variáveis.

\section{UMA REGRESSÃO ECONOMÉTRICA: MODELO DE VETCRES DE CORREÇÃO DE ERROS.}

Como podemos estimar o modelo esboçado anteriormente? Se as séries envolvidas no modelo fossem estacionárias, uma opção atraente consistiria na aplicação de Mínimos Quadrados Ordinários. Não obstante, se as séries não são estacionárias, os estimadores mínimos quadrados ordinários serão ineficientes. Portanto um passo inicial consiste em analisar as propriedades estocásticas das séries individuais.

A Figura 3 apresenta as trajetórias temporais das variáveis do modelo no período 1980:1/1989:12. Uma característica comum a todas as variáveis é dada pela existência de tendências seculares, onde, naturalmente, estas tendências possuem direções distintas. A existência de tendências nas séries sugere claramen- 
te processos não estacionários, o que é reafirmado pelos elevados coeficientes de autocorrelação para longos resíduos temporais. No domínio da frequiência, uma característica notável é a similitude dos cronogramas estimados com o espectro típico estudado por Granger (1966): em todos os casos - onde em menor média no caso do produto - os componentes de alta frequiência têm contribuição moderada, enquanto os componentes de períodos longos influem de forma predominante no comportamento das séries.

Formalmente, a Tabela 3 apresenta vários testes em relação à estrutura estocástica das séries individuais. No painel A, estatísticos alternativos submetem a prova a hipótese nula de que cada série contém uma raiz unitária contra a hipótese alternativa de que as séries são estacionárịas ao redor de uma tendência determinística. O painel $B$ submete a prova a hipótese de que as séries são voltas aleatórias sem drift contra a hipótese alternativa de que não é assim. Em geral, os resultados dos dois painéis indicam que a existência de raízes unitárias não pode ser ignorada. Finalmente, o painel $C$ parte da suposição de que não existe tendência determinística em nenhuma das variáveis - o qual é consistente com os testes anteriores - e então submete a prova hipótese de que as séries são voltas aleatórias sem drift, contra a hipótese de que as séries são voltas aieatórias com drift. Esta proposição é ignorada para o crédito e para a taxa de juros, mas não pode ser ignorada para $\mathrm{M} 1$ e para o produto. Isto torna recomendável a inclusão no modelo de um vetor de constantes, permitindo que os dados discriminem em quais equações tais constantes são significativas.

Em função dos resultados obtidos, a estimaçãodo modeloem sua especificação original não parece adequada. Em tal caso, um procedimento comum consiste em reformular o modelo usando as primeiras diferenças das variáveis, de modo que as séries usadas na estimação possam satisfazer as condições de estacionalidade.

Todavia, depois de um grande entusiasmo inicial, é agora reconhecido que a aplicação desta estratégia para a análise de variáveis não estacionárias poderia implicar custos onerosos, ao desconsiderar quase que totalmente as relações de longo prazo entre as variáveis. Em consequiência, a teoria econômica nos sugere frequiêntemente a existência de relações de equilíbrio entre deturminadas variáveis, entendendo, por isso, a tendência das forças de mercado a manter certas relações para períodos longos de tempo, apesar de inevitáveis desvios no curto prazo. Assim, quando as variáveis tendem a afastar-se de suas relações de equilíbrio, os mecanismos de mercado geram forças contrárias que tentam recuperar o equilíbrio. E é precisamente neste aspecto que a estratégia acima descrita representa um custo oneroso: ao diferenciar as variáveis, estamos ignorando quase toda a informação relevante em torno das relações de longo prazo entre as variáveis, limitando nosso conhecimento à dinâmica de curto prazo. 


\begin{tabular}{lcc}
\hline & A. $\Delta Y_{t}=\beta_{0}+\beta_{1} t+\beta_{2} Y_{t-1}+\sum_{t} \Delta Y_{i}$ \\
& $H_{0}:\left(\beta_{0}, \beta_{1}, \beta_{2}\right)=\left(\beta_{0}, 0,0\right)$ \\
\hline & $\theta_{3}{ }^{\text {(a) }}$ & $\tau_{\tau}=\tau_{\beta_{2}}{ }^{(b)}$ \\
M1 & 1,7889 & $-1,8888$ \\
Y & 4,0864 & $-2,7863$ \\
$\mathrm{R}$ & 2,9040 & $-2,2800$ \\
\hline
\end{tabular}

(B)

\begin{tabular}{|c|c|c|c|}
\hline \multicolumn{4}{|c|}{ B. $\begin{aligned} \Delta Y_{1}=\beta_{0}+\beta_{1} t+\beta_{2} Y_{t-1}+\sum_{t}^{P} \Delta Y_{i} \\
H_{0}:\left(\beta_{0}, \beta_{1}, \beta_{2}\right)=(0,0,0)\end{aligned}$} \\
\hline & $\theta_{2}^{(a)}$ & $\tau_{\alpha}=\tau_{\beta_{0}}^{(a)}$ & $\tau_{\alpha}=\tau_{\beta_{1}}{ }^{(a)}$ \\
\hline M1 & 1,7280 & 1,8487 & $-0,8820$ \\
\hline $\mathrm{C}$ & 4,0608 & $2,5635^{*}$ & $-1,0971$ \\
\hline $\mathrm{Y}$ & $0,7140^{*}$ & 2,2634 & 2,1066 \\
\hline $\mathrm{R}$ & $0,6402^{*}$ & $-0,9300$ & 1,0654 \\
\hline \multicolumn{4}{|c|}{ (C) } \\
\hline \multicolumn{4}{|c|}{ C. $\begin{array}{c}\Delta \mathrm{Y}_{\mathrm{t}}=\alpha+\beta \mathrm{Y}_{\mathrm{t}-1}+\sum_{\mathrm{i}}^{\mathrm{P}} \Delta \mathrm{Y}_{\mathrm{i}} \\
\mathrm{H}_{0}:(\alpha, \beta)=(0,0)\end{array}$} \\
\hline & $\theta_{1}^{(a)}$ & $\tau_{\alpha \mu}=\tau_{\beta_{0}}{ }^{(a)}$ & $\tau_{\mu}=\tau_{\beta_{1}}{ }^{(a)}$ \\
\hline M1 & 2,6832 & 1,6000 & $-1,6749$ \\
\hline $\mathrm{C}$ & $6,9060^{*}$ & $2,6279 * *$ & $-2,6376$ \\
\hline$Y$ & 0,7140 & 1,1600 & $-1,1535$ \\
\hline $\mathbf{R}$ & $0,1450^{*}$ & $-0,3809$ & $1,6576^{*}$ \\
\hline
\end{tabular}

(a) Os valores críticos estāo calculados em DICKEY \& FULLER (1981)

(b) Os valores críticos estão calculados em DICKEY \& FULLER (1979)

(1) O número do defasagens para a aplicação do teste ADF, p, foi escolhido, em cada caso, pelo Critério de Informação de Akaike.

* Significativo a $5 \%$

** Significativo a $10 \%$. 

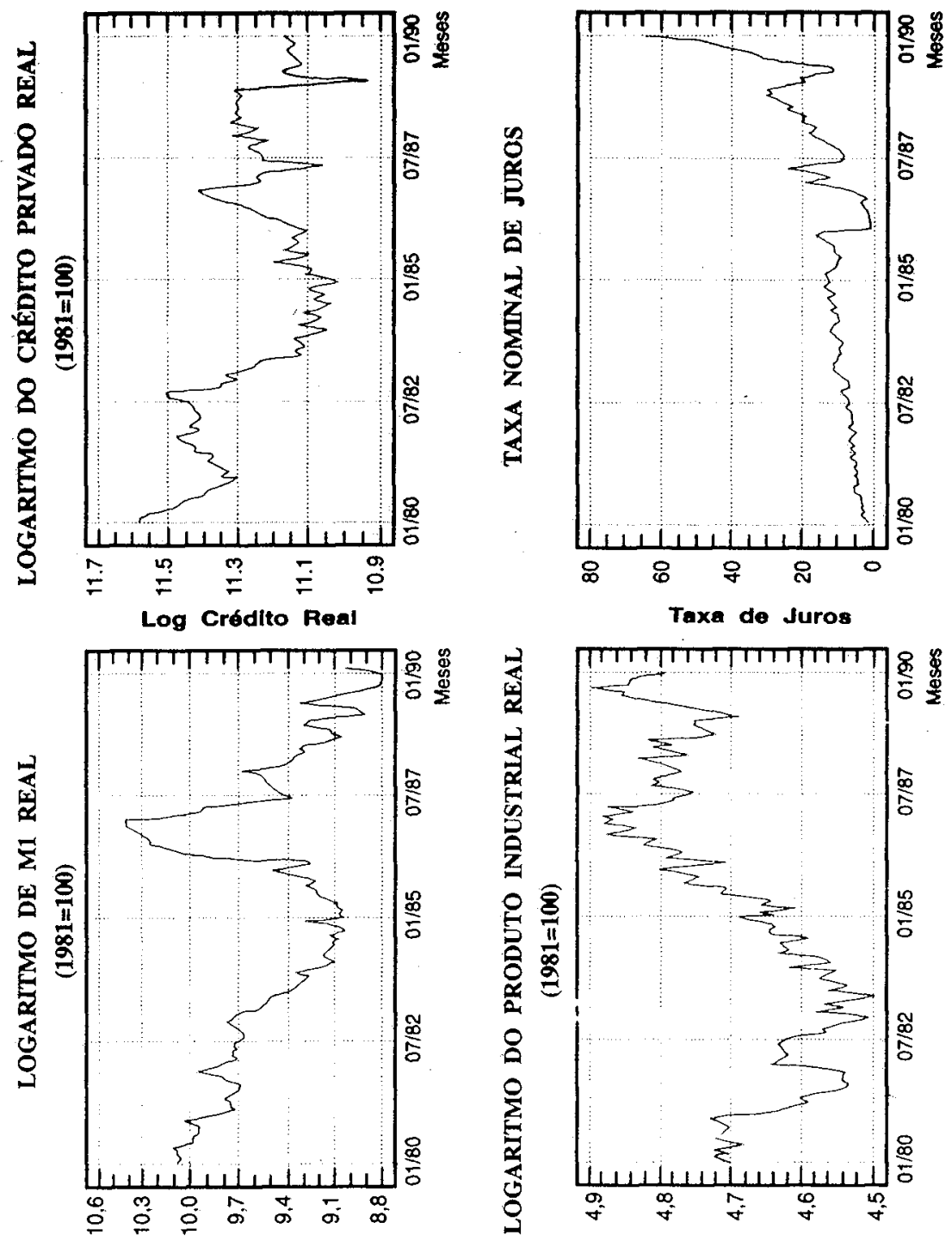

Figura 3 

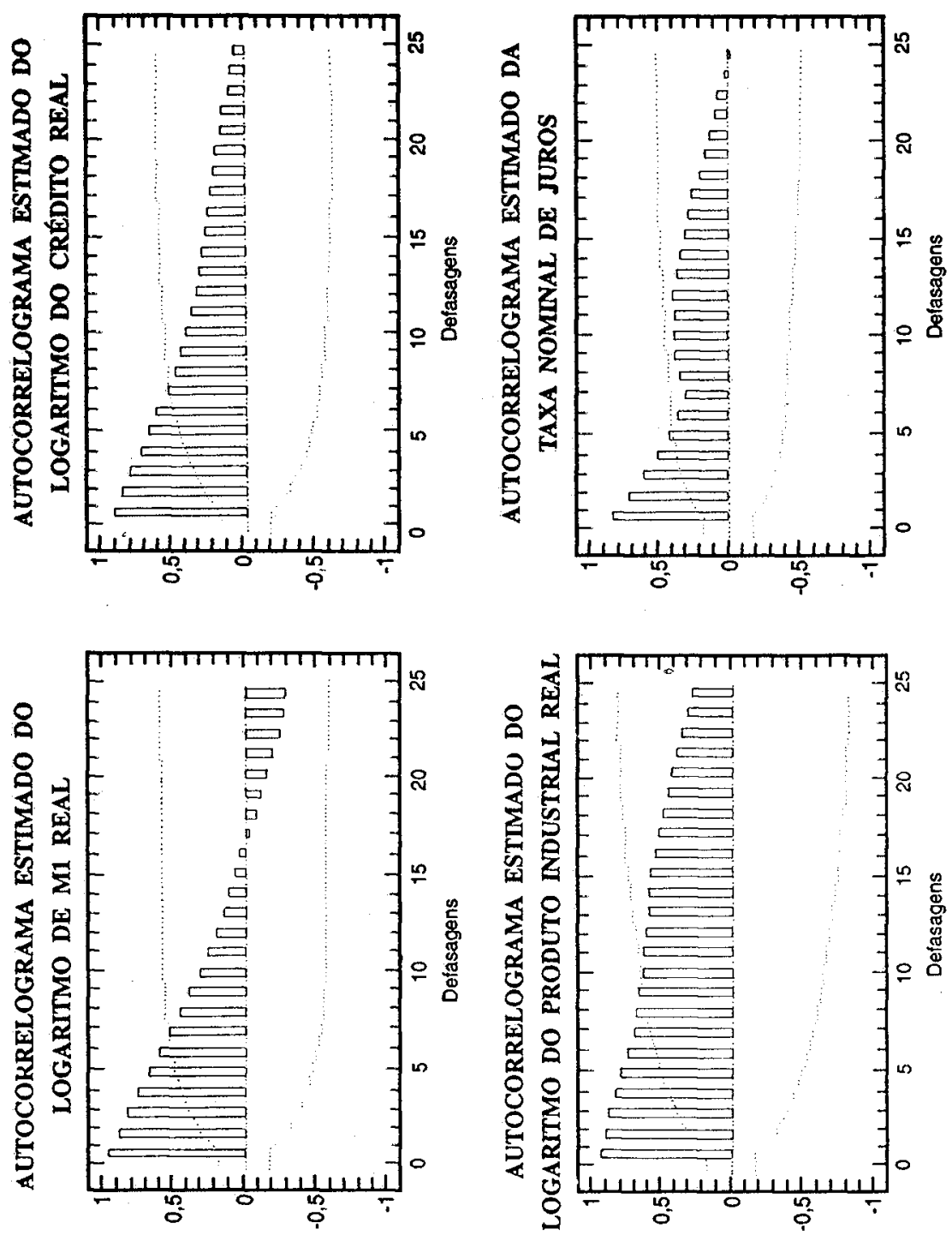

Figura 4 

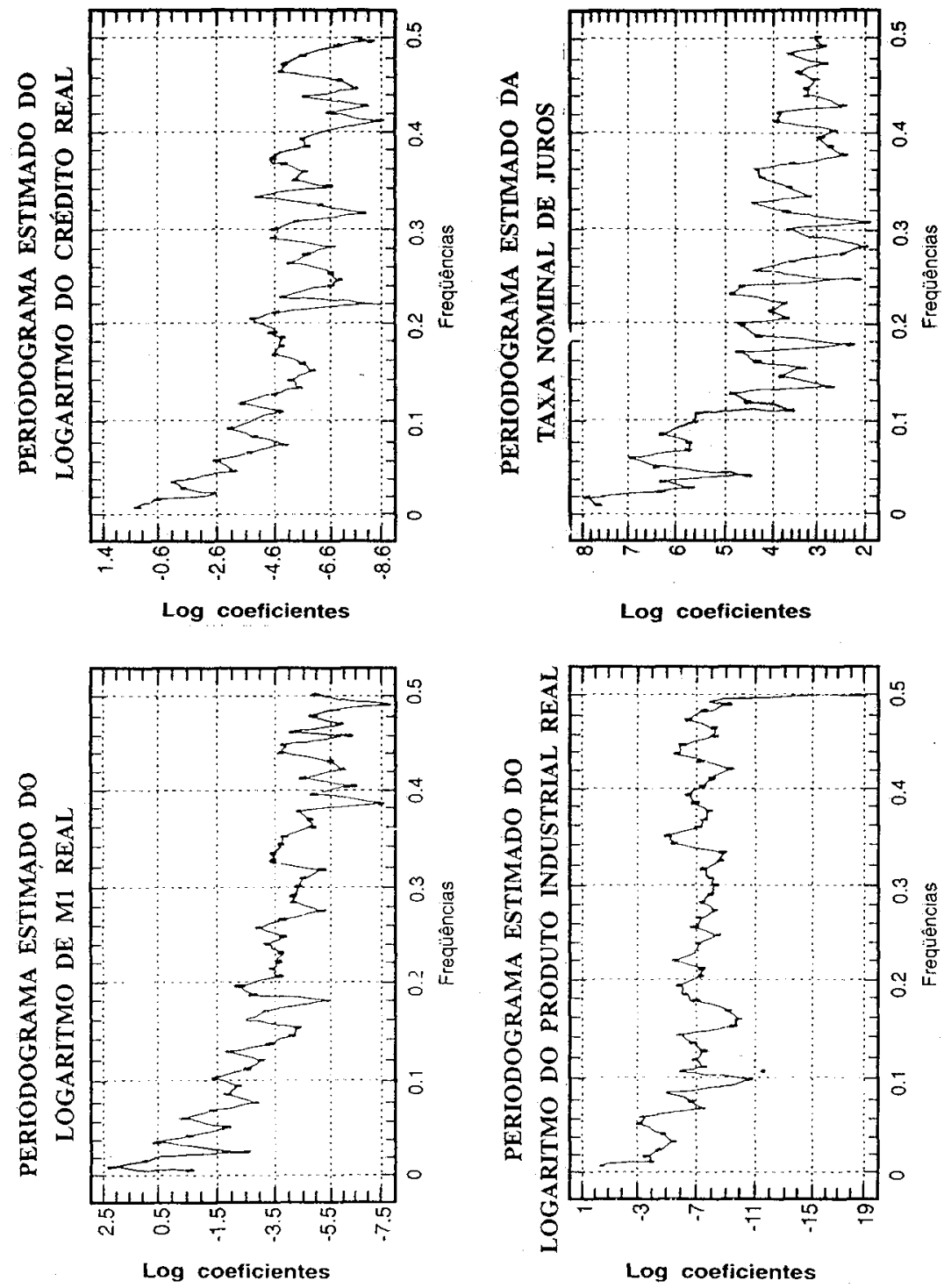

Figura 5

A análise de co-integração, atualmente em rápido desenvolvimento, nos permite a construção de modelos que capturam, mas não impõem, as relações de 
curto prazo entre as variáveis econômicas. Para introduzir esta análise, precisamos definir alguns conceitos.

DEFINIÇÃO. Uma variável aleatória, $\mathrm{X}_{\mathrm{t}}$, é denominada integral de ordem 0 , e denotada $X_{t}$ - I (0), quando seu espectro é finito, mas não zero, em todas as freqüências. Por extensão, uma variável aleatória, $\mathrm{X}_{\mathrm{t}}$ é denominada integral de ordem $k$, e denotada $\mathrm{X}_{\mathbf{t}} \sim \mathrm{I}(k)$, quando:

$$
\Delta X_{i}=(I-L)^{k} X_{i} \sim I(0)
$$

Consideremos agora um conjunto de variáveis, $\mathrm{W}_{1}, \ldots, \mathrm{W}_{\mathrm{n}}$, tal que $\mathrm{W}_{1}-\mathrm{I}(1)$ para $i=1, \ldots, n$. Em geral, uma combinação linear destas variáveis será também integral de ordem 1. Em alguns casos especiais, no entanto, as tendências das distintas variáveis poderiam cancelar-se uma à outra, gerando uma série integral de ordem 0 . Isto motiva a definição de variáveis co-integradas.

DEFINIÇÃO. Seja Y um vetor (coluna) de variáveis aleatórias integrais de ordem 1. As variáveis $\mathrm{Y}_{1}, \ldots, \mathrm{Y}_{\mathrm{n}}$ são denominadas co-integrais sempre que exista um vetor (fila) A, tal que

$$
z_{t}=A Y-I(0)
$$

Intuitivamente, duas variáveis são co-integrais quando não se separam sistematicamente de uma determinada relação. No contexto econômico, a relação $\mathrm{AY}=0$ pode ser vista como uma relação de equilíbrio, cujos desvios de curto prazo, representados por $z_{1}$ flutuam ao redor de um valor médio (não necessariamente igual a 0 ) sem manifestar tendência duradoura. $O$ vetor $A$ não precisa ser único e é denominado vetor de co-integração. Se reunimos estes vetores em uma matriz $\alpha$, a categoria desta matriz (isto é, o número de vetores de co-integração independentes) é denominada categoria de co-integração.

Consideremos novamente um vetor aleatório, $X_{t}$, integral de ordem 1 . Sem prejuízo quanto à generalização, assumamos que a esperança de seus incrementos é igual a 0 ; é dizer: $E\left[(1-L) X_{i}\right]=0$. Existe, portanto, uma representação multivariada de Wold:

$$
(I-L) X_{t}=C(L) a_{t}
$$

onde $\left(a_{\mathfrak{t}}\right)$ é uma seqüência de vetores aleatórios com a propriedade:

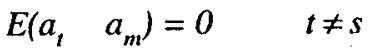

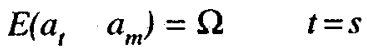

Após estas condições, é possível demonstrar o seguinte teorema: 


\section{TEOREMA 1. [(GRANGER \& ENGLE (1987)]}

Se um vetor aleatório, $X_{t}$, de dimensão $N x 1$ e satisfazendo as condições acima, é co-integrável, com categoria de integração igual a r, então:

(i) $\mathrm{C}(1)$ é de categoria $\mathrm{n}-\mathrm{r}$

(ii) Se existe uma representação de vetores auto-regressivos (VAR) com ordem finita, esta tenderá a forma:

$$
A(L) X_{t}=d(L) a_{t}
$$

onde $d(L)=1$ e $A(L)$ é uma matriz de polinômios finitos tal que: $A(0)=I_{n}$, e a categoria de $A(1)$ é igual a r.

(iii) Existe uma representação ARMA

$\mathrm{A}(\mathrm{L}) \mathrm{X}_{\mathrm{t}}=\mathrm{d}(\mathrm{L}) \mathrm{a}_{\mathrm{t}}$, com as propriedades: $\left.\mathrm{a}\right) \mathrm{d}(\mathrm{L})$ é um polinômio de defasagem escalar estável com $\mathrm{d}(1)<\infty ;$ b) $\mathrm{A}(0)=\mathrm{I}_{\mathrm{n}}$; c) $\mathrm{A}(1)$ tem categoria igual a $\mathrm{r}$.

(iv) Existem matrizes $\alpha$ e $\gamma$, de ordem nxr e rank $\mathrm{r}$, tais que:

$$
\begin{aligned}
& \alpha^{\prime} \mathrm{C}(1)=0 \\
& \mathrm{C}(1) \gamma=0 \\
& \mathrm{~A}(1)=\gamma \alpha^{\prime}
\end{aligned}
$$
forma:

(v) Existe uma representação de vetores de correção de erros (VEC), na

$$
\begin{aligned}
& A^{*}(L)(I-L) X,=-\gamma z_{t \cdot 1}+d(L) a_{t} \\
& A^{*}(O)=I_{n}
\end{aligned}
$$
rias.

donde $z_{t}=\alpha^{\prime} X_{t}$ é um vetor $r$-dimensional de variáveis aleatórias estacioná-

A proposição (i)é uma condição necessária e suficienie para a co-integração das variáveis e todas as demais proposições podem ser derivadas dela. ${ }^{7}$ Como é evidente, a representação de correção de erros é semelhante a um modelo de vetores auto-regressivos em diferenças: a distinção essencial é dada pelo termo $\mathrm{z}_{\mathrm{t}-1}$, o qual é uma combinação dos níveis das variáveis. $\mathrm{z}_{\mathrm{t}-1}$ mostra que se as

7 Ver GRANGER \& ENGLE (1987). 
variáveis são co-integráveis, o VAR em disparidade está mal especificado. Por sua vez, um VAR nos níveis das variáveis será assintoticamente consistente, mas ineficiente: isto é, se bem que as restrições sejam assintoticamente satisfeitas, sua imposição explícita deve produzir ganhos em termos de eficiência. Esta derivação é inteiramente confirmada por estudos de Monte Carlo, nos quais se mostra que os modelos VAR irrestritos (UVAR) são inferiores, em predições de médioe longo prazo, aos modelos de correção de erros. ${ }^{8}$

Assim, quando a teoria nos indica a possivel existência de relações de equilíbrio, os modelos de vetores com correção de erros representam um padrão de análise adequado. A pergunta imediata é: qual poderia ser o procedimento de estimação adequado para tais modelos? Como testar rigorosamente hipóteses relativas ao número de relações de longo prazo em um conjunto de variáveis? O método de máxima verossimilhança descrito a seguir responde a tais perguntas.

A estratégia consiste em formular a hipótese de co-integração como uma hipótese em relação ao nível de determinada matriz. Consideremos o modelo com $\mathrm{n}$ variáveis na forma:

$$
\mathbb{X}_{t}=\mu+\Pi_{1} \mathbb{X}_{t-1}+\ldots+\Pi_{k} \mathbb{X}_{t-k}+\hat{c}_{t}
$$

onde $\varepsilon_{1} \ldots \varepsilon_{1}$ são seqüências $\operatorname{IIN}_{n}(0, \Lambda)$ e $\mathbb{X}_{-k+1}, \ldots, X_{0}$ são fixos. Oproblema consiste em cstimar os $\left[n+k n^{2}+n(n+1) / 2\right]$ parâmetros correspondentes a $\left(\mu, \Pi_{1}, \ldots, \Pi_{k}, \Lambda\right)$ a partir de T observações das variáveis. Não é difícil verificar que a equação anterior pode também ser escrita na forma:

$$
\Delta \mathbb{X}_{t}=\mu+\Gamma_{1} \mathbb{X}_{t-1}+\ldots+\Gamma_{k-1} \Delta \mathbb{X}_{t-k+1}+\Pi \mathbb{X}_{t-k}+\varepsilon_{t}
$$

onde:

$$
\Gamma_{l}=-\left(I-\Pi_{l}-. .-\Pi_{i}\right) ; \quad \Pi=-\left(I-\Pi_{l}-. .-\Pi_{k}\right)
$$

A diferença entre este modelo e um VAR em diferenças consiste no termo $\Pi X_{t-k}$ : se as variáveis do vetor co-integram-se, a matriz $\Pi$ encerra informações sobre as relações de longo prazo. Existem três possibilidades em relação a esta matriz:

(I) nível $(\Pi)=0$. Neste caso, $\Pi$ é a maíriz nula e o modelo adequado é um VAR tradicional com diferenças.

(II) nível $(\Pi)=n$. Neste caso, o vetor $\chi_{t}$ é estacionário e o modelo adequado é um VAR tradicional nos níveis.

8 Ver ENGLE \& YOO (1987) para uma comparação dos resultados previśveis de ambos os tipos de modelo. 
(III) nível $(\Pi)=r<n$. Neste caso, existem duas matrizes $\alpha$ e $\beta$ de dimensão nxr, tais que $\Pi=\alpha \beta^{\prime}$.

Note-se que, no caso (3), $\beta^{\prime} \chi_{t}$ é estacionário mesmo que $\chi_{t}$, por si mesmo, não seja. Então, (15) pode ser interpretado como um modelo de correção de erros. A hipótese a testar é a hipótese de que existem r vetores de co-integração. É dizer:

(9)

$$
H_{0}: \Pi_{(n x n)}=\alpha_{(n x r)} \beta_{(n x r)^{\prime}}^{\prime}
$$

segundo Johansen \& Juselius, convém introduzir a notação:

$$
\begin{gathered}
\mathbb{Z}_{0 t}=\Delta X_{t} \\
\mathcal{Z}_{t}=\left[\Delta X_{t-1} \ldots \Delta X_{t-k-1} l\right]^{\prime} \\
\mathbb{Z}_{k t}=X_{t-k} \\
\Gamma=\left[\Gamma_{1} \ldots \Gamma_{k-1} \mu\right]
\end{gathered}
$$

de onde, como poderíamos verificar, $\mathbb{Z}_{1 \mathrm{t}}$ é um vetor de dimensão $[n(k-1)$ $+1]$, e $\Gamma_{t}$ é uma matriz de dimensão $n \times[n(k-1)+1]$. $O$ modelo (17) pode ser reescrito na forma simplificada:

$$
\mathbb{Z}_{0 t}=\Gamma \mathbb{Z}_{l t}+\Pi \mathbb{Z}_{k t}+\varepsilon_{t}(t=1, \ldots, T)
$$

Para um dado valor de $\Pi, \Gamma$ pode ser estimado pela regressão de $\left(\mathbb{Z}_{0_{t}}\right.$ $\Gamma \mathbb{Z}_{1 \mathrm{t}}$ ) contra $\Pi \mathbb{Z}_{\mathrm{k} \text { i }}$, dando o resultado:

$$
\Gamma=M_{01} M_{11}^{-1}-\Pi M_{k l} M_{11}^{-1}
$$

onde

$$
M_{i j}=\left(\Sigma \mathbb{Z}_{i t} \mathbb{Z}_{j t}{ }^{\prime}\right) / T \quad(i, j=0,1, k)
$$

Agora, nosso problema principal consiste em estimar $\Pi$ e seus fatores $\alpha$ e $\beta$, testando a hipótese de que $\mathrm{r}<\mathrm{n}$. Se denominamos como $\mathrm{R}_{\mathrm{ot}}$ e $\mathrm{R}_{\mathrm{kt}}$ os resíduos de uma regressão de $\Delta X_{t} \mathrm{e} \mathbb{Z}_{\mathrm{t}-\mathrm{k}}$ contra $\mathbb{Z}_{\mathrm{t}-\mathrm{l}}$, a função de máxima verossimilhança é dada por:

$$
L(\alpha, \beta, \Lambda)=|\Lambda|^{T / 2} \exp \left\{\sum_{t=l}\left(R_{0 t}-\Pi R_{k t}\right)^{\prime} \Lambda\left(R_{0 t}-\Pi R_{k t}\right) / 2\right\}
$$

Se $\beta$ fosse fixo, podemos minimizar em $\alpha$ e $\Lambda$ obtendo-se: 
(15)

$$
\begin{aligned}
& \hat{\alpha} ।_{\beta=\beta}=-S_{0 k} \bar{\beta}\left(\bar{\beta} ' S_{k k} \bar{\beta}\right)^{-1} \\
& \left.\hat{\Lambda}\right|_{\beta=\beta}=S_{00}-S_{o k} \bar{\beta}\left(\bar{\beta} S_{k k} \bar{\beta}\right)^{-1} \bar{\beta}^{\prime} S_{k o}
\end{aligned}
$$

onde

$$
S_{i j}=\left(\sum_{0}^{T} R_{1 t} R_{j t}^{\prime}\right) / T \quad(i, j=0, k)
$$

Com este elementos, é possível demonstrar o seguinte:

\section{TEOREMA 2. [JOHANSEN (1988): JOHANSEN \& JUSELIUS (1990)]}

Dada a hipótese $H_{0}: \Pi=\alpha \beta$, o estimador de máxima verossimilhança de $\beta$ é encontrado pelo seguinte procedimento:

(i) Resolver a equação:

$\left|\lambda S_{k k}-S_{k 0} S_{o o}^{-1} S_{o k}\right|=0$

o qual produz os autovalores $\lambda_{1}>\ldots>\lambda_{n}$ e a matriz de autovetores $V=\left(v_{1}\right.$, $\ldots, v_{n}$ ) normalizados de forma tal que $V^{\prime} S_{k k} V=I .^{9}$

(ii) Escolher $\beta$ como a matriz $\left(v_{1}, \ldots, v_{r}\right)$ que maximiza a expressão:

$L_{\max }^{-2 \pi}=\left|S_{00}\right| \sum_{i=1}^{r}\left(1-\lambda_{i}\right)$

(iii) Os estimadores de $\alpha$ e $\Lambda$ são encontrados introduzindo o estimador de $\beta$ nas expriessões (15).

(iv) Um teste de razão de semelhança para a hipótese $\mathrm{r}=\mathrm{n}$ contra a hipótese $\mathrm{r}<\mathrm{n}$ é dado por:

$$
Q_{1}=\cdot T \sum_{i=r+1}^{n} \ln \left(1-\lambda_{1}\right)
$$

9 Os autovetores deste prohlema não são convencionalmente calculados na maioria dos softwares. Contudo o problema pode ser transformado em um problema mais simples encontrando a matriz $\mathrm{C}$. al que $\mathrm{S}_{00}=\mathrm{C}^{*} \mathrm{C}$. Então, a problema convencional:

(I) $\quad\left|\lambda I \cdot C^{\prime \prime} s_{k 0} s_{00}^{-i} s_{o k} C^{\prime}\right|=0$,

nos dá os mesmos autovalores que o problema original. Por sua vez. os autovetores originais são calculados pela fórmula $C^{\prime} \beta^{*}$ onde $\beta^{*}$ e a soluçāo da equaçāo (I) 
(v) Um teste de razão de semelhança para a hipótese $r=m$ contra a hipótese $r=m+1$ é dada pọ:

$$
Q_{2}=-T \ln \left(1-\lambda_{m+1}\right)
$$

Assim, este teorema estabelece um teste adequado sobre o número de vetores de co-integração, oferecendo também um método de estimação para tais vetores. A distribuição assintótica das estatísticas $Q_{1} Q_{2}$ não é dada pela distribuição $\chi^{2}$, além de que corresponde a uma versão multivariada da distribuição Dickey-Fuller. Estas distribuições são descritas por determinadas integrais estocásticas e foram tabeladas, para alguns casos, em Johansen \& Juselius (1990).

\section{ESTIMAÇÃO DAS CONDIÇÕES DE EQUILÍBRIO:}

Retornemos agora ao modelo da equação (1). Através de algebrismos triviais, reformularemos o modelo na forma alternativa:

$$
\begin{gathered}
\Delta \mathbb{X}_{t}=\Gamma_{l} \Delta \mathbb{X}_{t-1}+\ldots+\Gamma_{l l} \Delta \mathbb{X}_{t-11}+\Pi \mathbb{X}_{t-12}+v D_{c t}+\xi D_{v t}+\mu+\varepsilon_{t} \\
E\left(\varepsilon_{t}\right)=0 \\
E\left(\varepsilon_{l} \varepsilon_{j}^{\prime}\right)=\left\{\begin{array}{cc}
\Sigma & i=j \\
0 & i \neq j
\end{array}\right.
\end{gathered}
$$

onde a relação entre as matrizes $\Pi_{1}, \Gamma_{1}$ e $\Pi$ é facilmente deduzida a partir da seção anterior. Obviamente, os vetores $\Delta \mathcal{X}_{t-i}(i=1, \ldots, 11)$, os quais representam primeiras diferenças das séries originais, são vetores estacionários. Deve também ser notado que o vetor de erros na equação (17), $\varepsilon_{t}$, é o mesmo que aparece em (1).

Agora, nosso objetivo imediato é testar a existência de determinadas relações de co-integração e (se a existência de tais relações é confirmada) estimar os vetores de co-integração correspondentes. Usando resultados prévios, a hipótese de co-integração pode ser expressa em termos de nívei dos fatores da matriz $\Pi$ (é dizer, $\left.\underset{(\text { nxrr })}{\alpha} e_{(n x r)}^{\beta}\right)$ pelo qual se torna adequado começar pela estimação desses fatores. $O$ estimador de Máxima Verossimilhança para a matriz $\beta$ é encontrado a partir dos autovetores na equação matricial:

$$
\left|\lambda S_{k k}-S_{k 0} S_{o o}^{-1} S_{o k}\right|=0
$$

onde as matrizes $S_{11}$ são convenientemente definidas para nosso caso particular. 
A Tabela 5 apresenta a solução deste problema para o caso da economia brasileira, usando a informação correspondente ao período $80: 1 / 89 ; 12 .{ }^{10}$ Os autovetores-solução formam uma matriz de dimensão $(4 \times 4)$, na qual $\hat{\beta}$ aparece como uma submatriz de dimensão (4xr). $O$ valor de $r$ deve ser determinado por meio das estatísticas $Q_{1} \in Q_{2}$. Consideremos primeiro a hipótese nula $r<=1$ versus a hipótese alternativa $r=0$. $O$ valor calculado de $Q_{1}$ é igual a 50,0 , superando amplamente o valor crítico correspondente. Testamos então a hipótese nula $\mathrm{r}<=$ 2. Neste caso, $Q_{1}$ ć igual a 16,74 e - dando um valor crítico de 17,84-, a hipótese nula não pode ser negada.

Finalmente, usamos $Q_{2}$ para reafirmar a decisão entre a hipótese nula $r=1$ contra a alternativa $r=2$. Neste caso, a hipótese nula é negada, ao comparar o valor calculado $\left(Q_{2}=33,24\right)$ com o valor crítico, $Q^{*}=21,28$. Em resumo, os testes de razão de semelhança indicam dois vetores de integração.

\section{TABELA 4}

\section{AUTOVALORES E AUTOVETORES}

\begin{tabular}{cccc}
\multicolumn{4}{c}{ Autovalores } \\
$\lambda_{1}$ & $\lambda_{2}$ & $\lambda_{3}$ & $\lambda_{4}$ \\
0,410 & 0,270 & 0,110 & 0,038
\end{tabular}

Autovetores

\begin{tabular}{|rr|rr}
\hline$-9,650$ & 9,260 & 9,070 & 13,530 \\
9,930 & $-22,630$ & $-7,910$ & $-9,240$ \\
9,400 & $-13,810$ & 4,420 & $-23,270$ \\
$-0,770$ & 0,200 & 0,230 & 0,680 \\
\hline
\end{tabular}

10 Neste modelo e na estimação posterior, a oferta monetária corresponde a MI segundo é definida pelo Banco Central. O crédito corresponde ao Crédito Total do Setor Privado, calculado pelo Banco Central. O Produto corresponde ao índice da Produção Industrial calculado pelo IBGE. Por último, a taxa de juro se refere a taxa de overnight calculada pela ANDIMA. Seguindo uma prática comum. as variáveis são expressas em logaritmos. Antes de extrair o logaritmo, as variáveis foram deflacionadas pelo índice de Preços ao Consumidor, usando como base o IPC médio de 1981 . 


\section{TABELA 5 \\ TESTES DE RAZÃO DE SEMELHANÇA \\ PARA DETERMINAÇÃO DAS DIMENSÕES DE $\beta^{(a)}$}

\begin{tabular}{ccrcr}
\hline HIPÓTESE & $\mathrm{Q}_{1}{ }^{(\mathrm{a})}$ & $\mathrm{Q}_{1}{ }^{\text {crit }}$ & $\mathrm{Q}_{2}{ }^{(\mathrm{a})}$ & \multicolumn{1}{c}{$\mathrm{Q}_{2}{ }^{\text {crit }}$} \\
\hline $\mathrm{r} \leq 3$ & & 8,08 & & 8,08 \\
$\mathrm{r} \leq 2$ & 16,74 & 17,84 & & 14,6 \\
$\mathrm{r} \leq 1$ & 50,00 & 31,26 & 33,74 & 21,28 \\
$\mathrm{r}=0$ & & 48,42 & & 27,34 \\
\hline
\end{tabular}

$\mathbf{n}$

(a) $Q_{1}=-T_{i=r+1} \sum_{i n} \ln \left(1-\lambda_{i}\right) ; Q_{2}=-T \ln \left(1-\lambda_{m+1}\right)$

(b) Os valores críticos aparecem tabelados em JOHANSEN \& JUSELIUS (1990).

Os vetores de co-integraçãoestimados podem ser normalizados de modoque o coeficiente da variável M1 seja igual a 1 . Assim, as relações de equilíbrio podem ser resumidas pelas equações:

$$
\begin{aligned}
& M I_{t}=1,02 * C_{t}+0,97 * Y_{t}-0,080 * R_{t} \\
& M I_{t}=2,44 * C_{t}+1,49 * Y_{t}-0,021 * R_{t}
\end{aligned}
$$

Dada a existência de mais de uma relação de equilíbrio no nosso sistema, a interpretação econômica das forças subjacentes não é imediata. No entanto um resultado notável é que na equação (19) a moeda e o produto real têm coeficientes quase idênticos em valor absoluto, o qual coincide com os resultados encontrados no Rei ǐu Unido (Hall, 1990), Dinamarca (Johansen/Juselius, 1990) e Finlândia (idem).

Se interpretamos a equação (19) como uma demanda per saldos reais, seus parâmetros parecem implicar uma condição de steady state com uma demanda por moeda de elasticidade unitária. Não obstante, esta inferência pode ser errada, dado que o segundo vetor estimado não satisfaz a mesma propriedade. Para garantir a existência de uma elasticidade unitária seria necessário que os dois vetores satisfizessem a mesma propriedade, o qual não é o caso. ${ }^{11}$

11 Uma "solução" tentadora é considerar que, na realidade, o segundo vetor não representa um vetor de cointegraçāo válido, tratando-se de um erro tipo I do teste. No entanto, esta "solução" não parece recomendável em vista da ampla margem pela qual foi negada a hipótese de somente um vetor de co-integração. 
Por último, as matrizes $\hat{\alpha}$ e $\hat{~} ̂$ são dadas pelas expressões:

$$
\begin{aligned}
\left.\alpha\right|_{\beta=\bar{\beta}}=-S_{0 k} \bar{\beta}\left(\bar{\beta} S_{k k} \bar{\beta}\right)^{-1} & \\
& {\left[\begin{array}{lr}
-0,006 & -0,008 \\
-0,017 & 0,000 \\
-0,008 & 0,007 \\
-1,200 & -0,820
\end{array}\right] }
\end{aligned}
$$

(22) $\hat{\Pi} ।_{\beta=\bar{\beta}}=\alpha \beta^{\prime}$

$$
=\left[\begin{array}{rrrr}
-0,020 & 0,173 & 0,057 & 0,003 \\
0,165 & -0,015 & -0,160 & 0,013 \\
0,145 & -0,172 & -0,176 & 0,008 \\
4,000 & 17,560 & 0,054 & 0,770
\end{array}\right]
$$

Depois de se obter $\hat{\Pi}$, a estimação das matrizes $\Gamma(i=1, \ldots, 11)$, e os vetores $v, \xi$ e $\mu$ é quase imediata. Só é necessário mudar o componente $\Pi \mathbb{X}_{1-12}$ para o lado esquerdo da expressão (17) e fazer então a regressão de $\left(\Delta X_{\mathrm{t}}-\hat{\Pi}^{\mathrm{l}} \mathcal{X}_{\mathrm{t}-12}\right)$ contra as variáveis $\Delta X_{\mathrm{t}-1}, \ldots, \Delta X_{\mathrm{t}-11}, \mathrm{D}_{\mathrm{c},}, \mathrm{D}_{\mathrm{vt}} \mathrm{e} 1$. No entanto os testes de significância indicam que alguns elementos não são estatisticamente significativos, pelo qual estimamos as matrizes e vetores mencionados com a restrição de que alguns componentes são nulos. As estimativas não são apresentadas no texto por carecerem de interesse particular.

\section{A DINÂMICA FORA DO EQUILÍBRIO}

O modelo pode agora ser usado para arialisar a dinâmica fora do equilíbrio. Em particular, estamos interessados em derivar as funções de impulso e a decomposição das variâncias das estimativas das variáveis. Um passo micial é transformar o modelo desde a forma (17), na qual foi estimado, aos parâmetros dados por (1). Naturalmente, a não-estacionalidade das séries impede uma representação de médias móveis nos níveis originais, pois as variáveis devem ser diferenciadas. Aplicando o operador (1-L) na estimação de (1), e ignorando os termos correspondentes às variáveis dummies, modelo estimado pode ser escrito na forma:

$$
\Delta \hat{\mathscr{X}}_{t}=\tilde{\Pi}_{1} \Delta X_{t-1}+\ldots+\tilde{\Pi}_{l 2} \Delta X_{t-12}+\varepsilon_{t}-\varepsilon_{t-1}
$$


onde:

$$
\hat{\Pi}_{1}=\hat{\Gamma}_{1}+I
$$

(26)

$$
\begin{aligned}
& \hat{\Pi}_{l}=\hat{\Gamma}_{1}-\hat{\Gamma}_{i-1} \\
& \hat{\Pi}_{12}=\hat{\Pi}-\hat{\Pi}_{11}
\end{aligned}
$$

Dada a presença do termo $\varepsilon_{\mathrm{t}-1}$, esta representação não corresponde a um Vetor Auto-regressivo (VAR), senão, de melhor maneira a um Vetor Autoregressivo-Médias Móveis (VARMA). A decomposição da variância de estimação pode ser rapidamente derivada. Por substituições sucessivas na expressão anterior, chegamos na expressão:

$$
\Delta \hat{X}_{t}=\hat{M}_{o} \varepsilon_{t}+\hat{M}_{1} \varepsilon_{t-1}+\hat{M}_{2} \varepsilon_{t-2}+\ldots
$$

onde

$$
\begin{gathered}
\hat{M}_{0}=I \\
M_{l}=I+\Pi_{1} \\
\hat{M}_{I}=\sum_{s=1}^{(I, I 2)} \hat{\Pi}_{s} \hat{M}_{i-s}(s \geq 2)
\end{gathered}
$$

Finalmente, se denotarmos por $\mathrm{S}$ a decomposta de Choleski da matriz de variâncias-covarâncias de $\varepsilon_{1}$ (é dizer, $\Sigma=S S$, sendo $S$ triangular inferior), e definimos $u_{t}$ como um vetor de perturbações tal que $\varepsilon_{\mathrm{t}}=S u_{\mathrm{t}}$ a expressão se transforma em:

$$
\Delta \hat{X}_{t}=\hat{M}_{M} S u_{t}+\hat{M}_{1} S u_{t-1}+\hat{M}_{2} S u_{t-2}+\ldots
$$

cujas perturbações, $u_{t}$, são ortogonais e, por conseguinte, as funções de impulso podem ser, então, calculadas. Os resultados numéricos deste exercício são detalhados em Guzman, 1991. Para nossos fins, são especialmente relevantes as funções de impulso da moeda e o crédito sobre o produto real, reproduzidas nas figùı as 6 e 7. As funções de impulso revelam o modo como um choque estocástico em uma variável afeta as demais variáveis dc médio em cada período futuro, o qual permite conhecer o estado do sistema a diferentes limites de tempo. 


\section{FIGURA 6 \\ RESPOSTA DINÂMICA DO PRODUTO \\ A UM DESVIO PADRÃO EM Um}

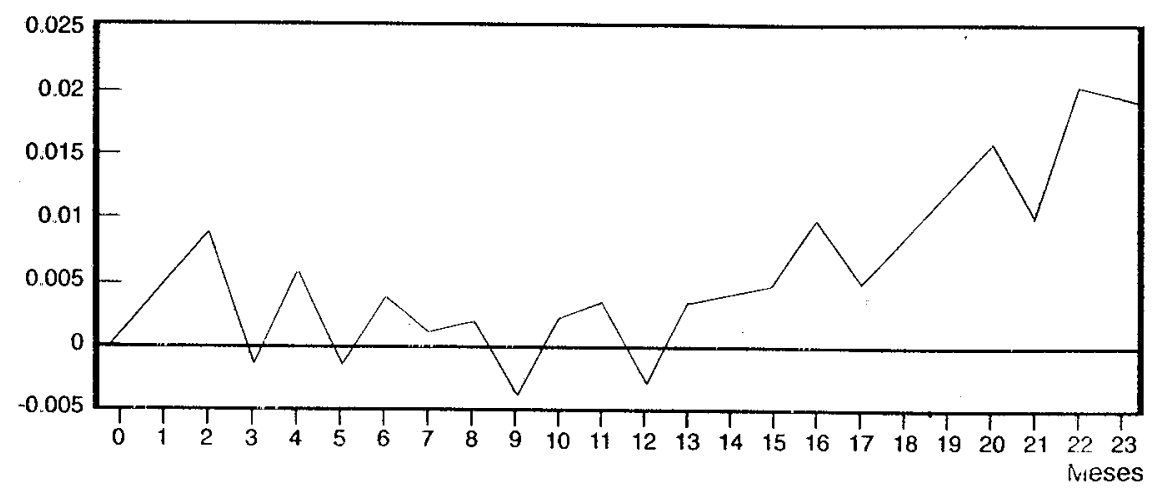

FIGURA 7

RESPOSTA DINÂMICA DO PRODUTO

A UM DESVIO PADRÃO EM Uc

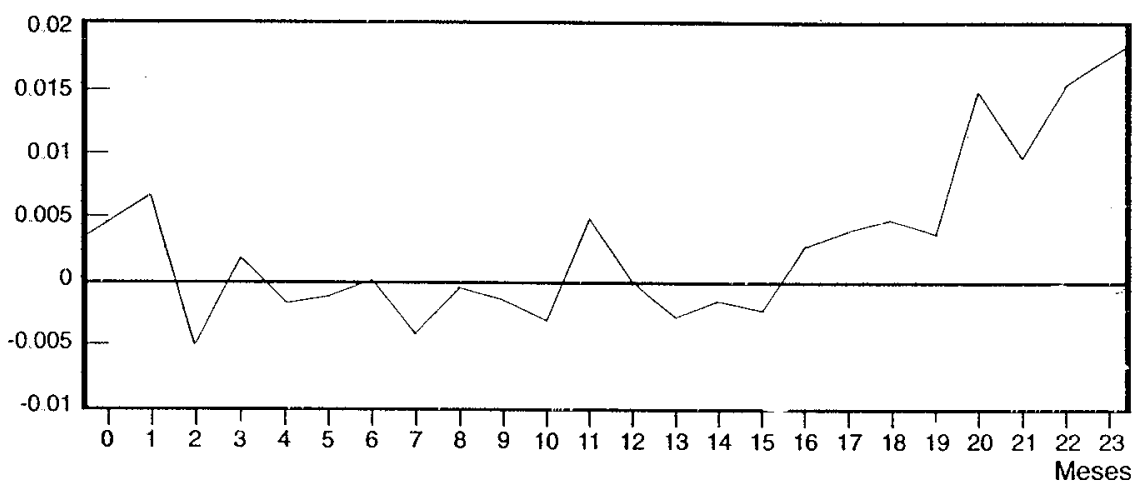

As duas funções de impulso apresentam um comportamento cíclico nos primeiros períodos. Naturalmente, esta característica resulta previsível: dada a natureza VARMA da representação(25), os padrões de impulsodeveriam ser mais complexos que os encontrados normalmente em modelos auto-regressivos.

Em termos gerais, os efeitos sobre o produto dos choques monetários e creditícios são quase semelhantes, ainda que com alguma diferenças no padrão temporal. Uma distinção perceptível é que os efeitos dos choques monetários se revelam mais rapidamente que os choques de origem creditícia. Assim, por exemplo, os efeitos do crédito só se manifestam de forma sustentada a partir do 
décimo-sexto periodo, enquanto que os choques monetários iniciam sua tendência sustentada a partir do décimo-terceiro mês.

Um resultado distinto emerge a partir da decomposição da variância das predições do produto real. Neste caso, a supremacia dos efeitos monetários tornase evidente. Desde os primeiros períodos, a porção da variância de previsão do produto explicada pelos choques monetários supera de forma notável a porção explicada pelos choques creditícios. Este resultado se faz, todavia, mais evidente à medida que avançamos no tempo: finalmente no $24^{\circ}$ período os choques em M1 explicam os $30 \%$ da variância de previsão, enquanto que os choques creditícios explicam somente ao redor de $18 \%$.

\section{FIGURA 8 \\ DECOMPOSIÇÃO DA VARIÂNCIA \\ DA PREVISÃO DO PRODUTO REAL}

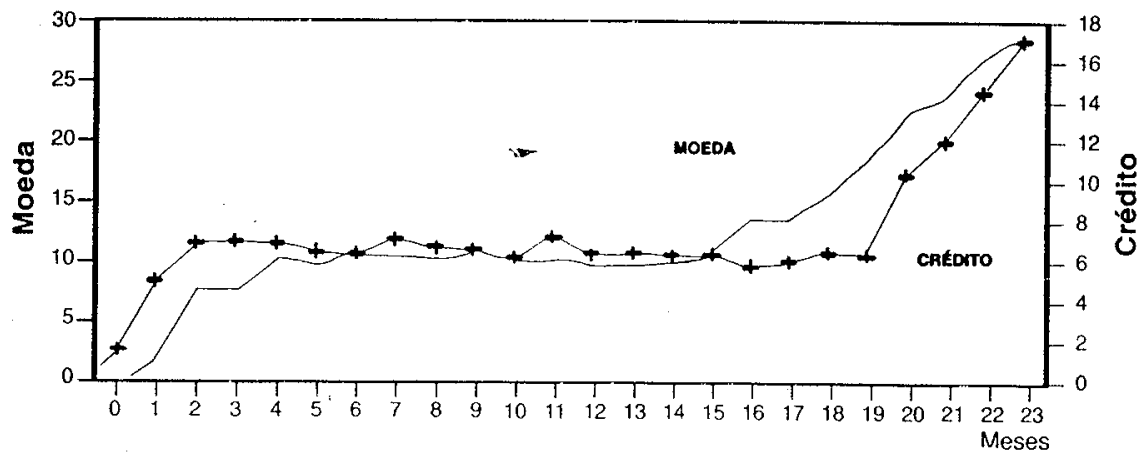

Nossos resultados, em conjunto, não são certamente confortáveis para as teorias que concedem um poder especial para as perturbações creditícias. Todavia quando os choques de crédito desempenham um papel importante, este papel não pareci alcançar a dimensão que deveria esperar-sc à luz de tais teorias. Pelo contrário, os resultados reafirmam a relevância particular - tradicionalmente percebida mas nunca claramente entendida - dos choques de caráter monetário.

\section{CONCLUSC̃ES}

Agora, os resultados obtidos através do modelo estimado podem ser brevemente resumidos da seguinte forma:

a) As variáveis incorporadas ao modelo são todas co-integráveis, com um nível de co-integração igual a 2 . Concretamente, isto significa que, no longo prazo, estas variáveis não se afastam sistematicamente do espaço gerado pelas relações: 


$$
\begin{aligned}
& M I_{t}=1,02 * C_{t}+0,97 * Y_{t}-0,080 * R_{t} \\
& M I_{t}=2,44 * C_{t}+1,49 * Y_{t}-0,021 * R_{t}
\end{aligned}
$$

Assim, estas relações podem ser entendidas como condições de equilíbriono longo prazo.

b) Os efeitos dos choques monetários e choques creditícios, ao longo do tempo, são relativamente semelhantes. Em ambos os casos, o efeito final é positivo. Contudo os impulsos dos choques monetários se manifestam mais rapidamente que os impulsos dos choques creditícios.

c) A variância das previsões do produto é fundamentalmente explicada pelos choques de caráter monetário e, naturalmente, por choques no próprio produto. A porção dessa variância atribuída aos choques creditícios é relativamente reduzida.

d) Em geral, os resultados obtidos não confirmam as teorias segundo as quai i as perturbações creditícias explicam de forma fundamental o comportamento do produto. Todavia, quando tais perturbações possuem certamente efeitos importantes, tal importância parece ter sido exagerăda em alguns estudos de natureza teórica.

\section{BIBLIOGRAFIA}

BERNANKE, Ben S Nonmonetary Effects of the Financial Crisis in the Propagantion of the Great Depression American Economic Review, v. 73, n. 3. p 257.76. June, 1983

BRANDĀO, A. C. Moedae Preços Relativos: A Evidência Empirica Revista de Econometria, 1985, v.8, n 1.p. 33 . 80.

CAIADO. Elcyon \& ROSSI, Josse Fontes Fxógenas de Aceleraçãc Inftacionária no Brasil Rio de Janeiro: IPEA. 1991. (Texto para discussão n²16)

CARDOSO. Eliana Uma Equação para a Dcmarda de Mocda no Brasil Pesquisa e Planejamento Fconômico, $v$.11, n. 3, p. 617-35, 1981

CARNEIRO NETC, Dionísio \& FRAtiA NFTO. A Variáveis de Crédito e Endogeneidade dos Agregados Monetários: Nota Sobre a Evidência Empirica nos Anos 70. Pesquisa e Planejamento Econômico, v. 14, n 1. p.175-96, 1984

CHOW. Gregory. Econometrics New York : McGraw.Hill. 1983.

Dynamic Control of Eccnometric Models New York: McGraw-Hill, 1981

. Analysis aud Control of Dynamic Systems. New York: Mcgraw-Hill. 1975.

DICKEY, David D, \& FULLER, Wayne A Likelihood Ratio Statistics for Autoregressive Time Series with Unit Root. Econometrica. v. 49, n 4, p 1057-72, 1981.

Distribution of the Estimators for Autoregressive Time Series with a Unit Root. Journal of the Americam Stutiscal Association, $v_{*}$ 74, n 366, p 427-31, 1979

ENGLE, Robert F. \& YOO, Byung S. Forecasting and Testing in Co-integrated System. Journal of Econometries, 35, p.143-59. 1987.

\& GRANGER, C. W. J. Cointegration and Error Correction: Representation. Estimation and Testing. Econometrica, v.55, n.2, p.251-76, 1987. 
FISHER, Irving. The Debt-Deflation Theory of Great Depressions. Econometria, v.1, n.1, p.337-57, 1933.

(1923) The Purchasing Power of Money. Citado em: SPENCER, Roger W. Channels of Monetary Influence: A Survey. Federal Reserve Bank of St. Louis Review, p.8-26, nov. 1974

FUL.L.ER, Wayne A. Introdution to Statistical Time Series. New York: Willey Ed., 1976.

GODFIELD, S. W. The Case of Missing Money. Brookings Papers on Economic Activity, 1976.

GRANGER, C. W. J. Developments in the Study of Cointegrated Economic Variables. Oxford Bulletin of Economics and Statistics, v. 48, n.3, p. 213-28, 1986.

. The Typical Spectral Shape of an Economic Variable. Econometrica, v.34, n.1. p.150-61, 1966.

1974 \& NEWBOLD, Paul. Spurious Regressions in Econometrics. Journal of Econometrics, n. 2. p.111-20,

. \& NEWBOL.D, Paul. Forecasting Economic Time Series. New York: Academic Press, 1977.

GURLEY, John G. \& SHAW, E. S Financial Aspects of Economic Development. American Economic Review, v.45, n.4, p.515-38, 1955.

GUZMAN, Rolando M Moeda e Crédito na Economia Brasileira: Um Modelo com Vetores de Correção de Erros. Tese de Mestrado, Universidade Federal do Rio Grande do Sul, 1991.

Moneda en la Teoria Economica. Mimeo, não publicado, 1990.

HALL. S. G. Maximum L.ikelihood Estimation of Cointegration Vectors. Oxford Bulletin of Economics and Statistics, v.51, n.2, p. 213-18, 1990.

HENDRY, David F. Econometric Modelling with Cointegrated Variables: An Overview. Oxford Bulletin of Economics and Statistics, v.48, n.3, p.201-12, 1986.

JOHANSEN, Soren. Statistical Analysis of Cointegration Vectors. Journal of Dynamics and Control, n. 12, 1988.

\& JUSELJUS, Katarina. Maximum Likelihood Estimation and Inference on Cointegration - With Applications to Demand for Money. Oxford Bulletin of Economics and Statistics, v.52, n.2, p. 169-200, 1990.

JUDGE, G. G. et alii. Introduction to the Theory and Practice of Econometrics. 2nd ed. New York: Willey, 1987.

. The Theory and Practice of Econometrics. New York: Willey, 1985.

LITTERMAN, R \& WEISS, L. Money, Interest Rates, and Output: A Reinterpretation of Postwar U.S. Data. Econometrica, v.53, n 1, p 129-56, 1985

LUCAS, Robert Econometric Policy Evaluation: A Critique. Carnegie-Rochester Conference Series on Public Policy. p. 19-46, 1976.

MISHKIN, Frederic S. Illiquidity, Consumer Durable Expenditure, and Monetar; Policy American Fconomic Review, v.66, n 4, p 642-53, 1976

NELSON. Charles R. \& PLOSSER, Charles I. Trends and Random Walk in Macroeconomic Time Series. Journal of Monetary Economics, n. 10. p.139-62. 1982.

PEREIRA, Pedro L.. Vals. Co-Integração e suas Representações. Revista Brasileira de Econometria p. 293-310, 1990.

POOL.E. William Optimal Choice of Monetary Policy Instruments in a Simple Stochastic Macro Model Quarterly Journal of Fconomics, p. 197-216. May 1970

Monetary Policy Lessons of Recent Inflation and Desenflation. Journal of Economic Perpectives, v. 2, n.3.p. $7.3-100,1988$

ROBERTSON, John \& ORDEN, David. Cointegration and Long-Run Monetary Neutrality: A Vector ErrorCorrection Model of Money and Prices in New Zealand. Texto apresentado na AAEA Meetings, Tennessee.

ROSSI, Jose. A Demanda por Moeda no Brasil: O que ocorreu a partir de 1980? Pesquisa e Planejamento Econômico, v. 18, n.1, p. 37-54. 1988

SCADDING, John \& JUDD, John. The Search for a Stable Money Demand Function: A Survey of the Post-1973 Literature. Journal of Economic Literature, v. 20, p.993-1023, 1988.

STOCK. James H. \& WATSON. Mark W. Variable Tnds in economic Time Series. Journal of Economic Perspectives, v.2, n. 3, p. 147.74, 1988. 
SDMS, C. Macrocconomics and Reality. Econometrica, v.48, n.1, p.1-48, 1980.

. Money, Income, and Casuality. American Economic Review, v.62, p.540-52, 1972.

SPANOS, Aris. Foundations of Econometric Modelling. Cambridge: Cambridge University Press, 1986.

\section{ABSTRACT \\ MONEY AND CREDIT IN THE BRAZILIAN ECONOMY: A MODEL WITH ERROR CORRECTION VECTORS}

This article searches for the relation between money, credit and aggregated product in the Brazilian experience during the $1980 / 1989$ period. A key element of the study is the estiamtion of Errors Correction Vectors Model which allows the identification of relations between variables in the short and long terms, in opposition to conventional methodologies which analyze only shorterm relations. The text starts with the stocastic properties of the different series, paying special attention to its integration orders. Then de model is estimaded through the method of maximum similarity outlined by Johansen (1989) and Johansen/Juselius (1990). At the end, the estimation is used for analyzing the time pattem of impulses and answers between the variables. A relevant outcome is that changes of product have been caused mainly by monetary shocks while the credit shorks exerted a smaller influence. 